\title{
Effect of Spherical, Rod-Like and Chain-Like Magnetic Nanoparticles on Magneto-Optical Response of Nematics
}

\author{
P. BurY ${ }^{a, *}$, M. VeveričíKK ${ }^{a}$, P. KopČAnskÝ ${ }^{b}$, M. Timko ${ }^{b}$ And V. ZÁViŠOVÁ ${ }^{b}$ \\ ${ }^{a}$ Department of Physics, Žilina University, Univerzitná 1, 01026 Žilina, Slovakia \\ ${ }^{b}$ Institute of Experimental Physics, Slovak Academy of Sciences, Watsonova 47, 04001 Košice, Slovakia \\ (Received July 31, 2018; revised version March 29, 2019; in final form July 16, 2019)

\begin{abstract}
The effect of magnetic nanoparticles of low concentrations and different shapes, including spherical, rodlike, and chain-like shape on the magneto-optical response of nematic liquid crystal 6CHBT is investigated. The magneto-optical effect was examined using a linearly polarized laser beam $(532 \mathrm{~nm})$ passed through liquid crystal cell under both linearly increasing and pulsed magnetic fields. The analysis of observed magneto-optical characteristics and magneto-optical time responses confirmed the role of both concentration and shape of magnetic particles on the resultant magneto-optical behavior concerning both its stability and switching time.
\end{abstract}

DOI: 10.12693/APhysPolA.136.101

PACS/topics: liquid crystal, magnetic particles, ferronematics, magneto-optical response

\section{Introduction}

Ferronematics are magnetically active anisotropic fluids which are a manifestation of the idea of Brochard and de Gennes [1], who suggested that doping liquid crystals (LCs) by fine magnetic particles may enhance their sensitivity to magnetic fields. Experiments on ferronematics confirmed, on the one hand, that magnetic particles considerably affected the behavior in external fields, and on the other hand, they proved that the interaction between the host liquid crystal and the magnetic particles was more complex than that assumed initially [2-5]. LCs can be generally oriented under electric or magnetic fields due to the anisotropy of dielectric permittivity or diamagnetic susceptibility [6]. As the former is of the order of unity in conventional devices the driving voltages are of the order of a few volts. However, due to the small value of the anisotropy of the diamagnetic susceptibility, the magnetic field necessary to align LCs must reach rather large values $(B>1 \mathrm{~T})$. Doping them with a small amount of magnetic particles just enhances the LCs magnetic susceptibility [1]. The small volume concentration of magnetic particles ensures that the inter-particle magnetic dipole-dipole interaction can be ignored. It can be expected then that the coupling between $\boldsymbol{m}$ and $\boldsymbol{n}$ under the applied magnetic field should result in the reorientation of $\boldsymbol{n}[3,4,7]$. Here the unit vector $\boldsymbol{n}$ (the director) describes the preferential direction of the nematic molecules and the unit vector $\boldsymbol{m}$ denotes the orientation of the magnetic moment of the magnetic particles.

It was shown that the addition of particles to liquid crystals, except for the reduction of the Fréedericksz

*corresponding author; e-mail: peter.bury@fel.uniza.sk transition driving electric and/or magnetic field, can also improve optical properties including the switching behavior of the pure LC [8-10]. The optical, mostly electrooptical, techniques were used to study orientational transitions in nematic LCs doped with ferroelectric nanoparticles [9], carbon nanotubes [8], or arranged on polymer surfaces [11]. It was found that the field assisted orientational variation can be accompanied by light intensity oscillations that could be attributed to the redistribution of the nematic director $\boldsymbol{n}$ from uniform alignment [11, 12]. The magneto-optical effect was investigated in lyotropic LCs doped with ferrofluid in their isotropic phase [13, 14] and also in nematic LCs doped with ferromagnetic particles [15-17]. The results obtained showed, except for the reduction of the Fréedericksz transition driving magnetic field, that the optical activity of LCs induced by ferromagnetic dopant results in the elliptical polarization of the emerging linearly polarized light beam with increase of magnetic field due to the distortions of the nematic order by magnetic particles. The decrease of magnetic threshold in such LC suspensions is the result of magnetic properties of ferromagnetic particles only. The magneto-optical response of ferronematic LCs was also used as the principle of visualization of magnetic fields [18].

Recently, we have studied the effect of magnetic particles on LC structural changes in electric and magnetic fields using the measurement of attenuation of the surface acoustic wave (SAW) propagating along ferronematic liquid crystal. The magnetic nanoparticles in low volume concentration $\left(\Phi=10^{-4}-10^{-5}\right.$ ) and a different arrangement, including spherical, rod-like, and chainlike shape, as a dopant in the LC (6CHTB), were investigated [19-21]. The structural changes of these ferronematics were studied also by capacitance measurements [22-24]. Results of both experimental investigations showed the significant influence of shape (size) 
and concentration of magnetic particles on the threshold fields and indicated soft anchoring in the case of spherical magnetic particles and rigid anchoring in the case of rod-like magnetic particles, with parallel initial orientation. In this work, we report results of magnetooptical investigations of these nematic LCs doped with magnetic particles of different arrangements and concentrations with the aim to reveal their optical properties regarding their structural changes occurring under magnetic field.

\section{Experimental details}

The ferronematic samples studied were based on the thermotropic nematic 4-(trans-4'-n-hexylcyclohexyl)isothiocyanatobenzene (6CHBT). 6CHBT is a lowmelting enantiotropic liquid crystal with high chemical stability [24]. The temperature of the nematic-toisotropic transition (clearing point) of the studied nematic is $T_{N I}=42.8^{\circ} \mathrm{C}$. The samples of nematic suspensions doped with magnetic particles of various arrangements, including spherical, rod-like, and chain-like shape were prepared by specific procedures [5]. The doping was simply done by adding this suspension, under continuous stirring, to the liquid crystal in the isotropic phase. Due to the small volume concentrations of the magnetic particles $\left(\Phi=1 \times 10^{-5}, 5 \times 10^{-5}, 1 \times 10^{-4}\right)$ and surfactant in the prepared ferronematic samples, the interparticle dipole-dipole interactions can be avoided.

The synthesis of spherical magnetic particles was based on the co-precipitation of $\mathrm{Fe}^{2+}$ and $\mathrm{Fe}^{3+}$ salts by $\mathrm{NH}_{4} \mathrm{OH}$ followed by dissolution of $\mathrm{FeSO}_{4} \cdot 7 \mathrm{H}_{2} \mathrm{O}$ and $\mathrm{FeCl}_{3} \cdot 6 \mathrm{H}_{2} \mathrm{O}$ in deionized water and then heating to $60^{\circ} \mathrm{C}$ to obtain $\mathrm{Fe}_{3} \mathrm{O}_{4}$ precipitate $[23,24]$. The mean diameter of the spherical magnetic particles was $11.6 \mathrm{~nm}$. Rod-like magnetic particles were synthesized through hydrolysis of $\mathrm{FeCl}_{3}$ and $\mathrm{FeSO}_{4}$ solutions (the $\mathrm{Fe}^{3+}$ to $\mathrm{Fe}^{2+}$ molar ratio was $2: 1$ ) containing urea. In a typical experiment $\mathrm{FeCl}_{3} \cdot 6 \mathrm{H}_{2} \mathrm{O}, \mathrm{FeSO}_{4} \cdot 7 \mathrm{H}_{2} \mathrm{O}$, and $\left(\mathrm{NH}_{2}\right)_{2} \mathrm{CO}$ were all dissolved in purified deoxygenated water. This mixture was added to a flask with a reflux condenser and heated in a water bath for $12 \mathrm{~h}$ at $90-95^{\circ} \mathrm{C}$. A dark precipitate was observed to have formed. The average diameter of rod-like particles was $80 \mathrm{~nm}$ and the mean length determined from the histogram of the size distribution was $1200 \mathrm{~nm}[22,23]$. The chain-like particles were obtained from the magnetotactic bacterium Magnetotacticum magnespirillum (AMB-1) [23]. The mean size of a single magnetic particle coated with a surfactant, i.e., of the magnetosome, was $34 \mathrm{~nm}$, and therefore, the mean length of the chainlike particles was $446 \mathrm{~nm}$. In all cases the size of the magnetic particles was much greater than the dimensions of the liquid crystal molecules, i.e., the magnetic particles could be regarded as macroscopic objects floating in the liquid crystal. Some magnetic properties of investigated suspensions such as the density of anchoring energy $W_{s}$ or characteristic parameter $\omega$ were determined in previous works [22, 23].
Magneto-optical investigations were done in the arrangement shown in Fig. 1. The LC cell holder was placed in the middle of electromagnet, between poles. The LC cells were prepared from two float glasses of thickness $0.7 \mathrm{~mm}$, with ITO transparent conductive layers, separated by $50 \mu \mathrm{m}$, and alignment layers rubbed in a parallel direction to electrodes to promote the parallel alignment. The semiconductor laser beam (532 nm, $5 \mathrm{~mW}$ ) was normally incident on the cell's glass using optical triangular prism and polarizer. The intensity of transmitted light was recorded by means of a photodetector connected to a multimeter and subsequently to the computer that monitored the light intensity as a function of magnetic field. The magnetic field that was controlled also by computer could be increased/decreased continuously ( $80 \mathrm{mT} / \mathrm{min})$ or altered by a jump (pulsed change) with the relaxation time of the increase/decrease shorter than $0.3 \mathrm{~s}$. The pulse width (duration) $120 \mathrm{~s}$ was chosen on the basis of our previous results obtained investigating structural changes in these LCs using surface acoustic waves [19, 20, 21] when SAW changes could reach the saturated state during such long time. No bias magnetic field was applied before starting the measurement so that suspensions could appear to be compensated ferronematics.

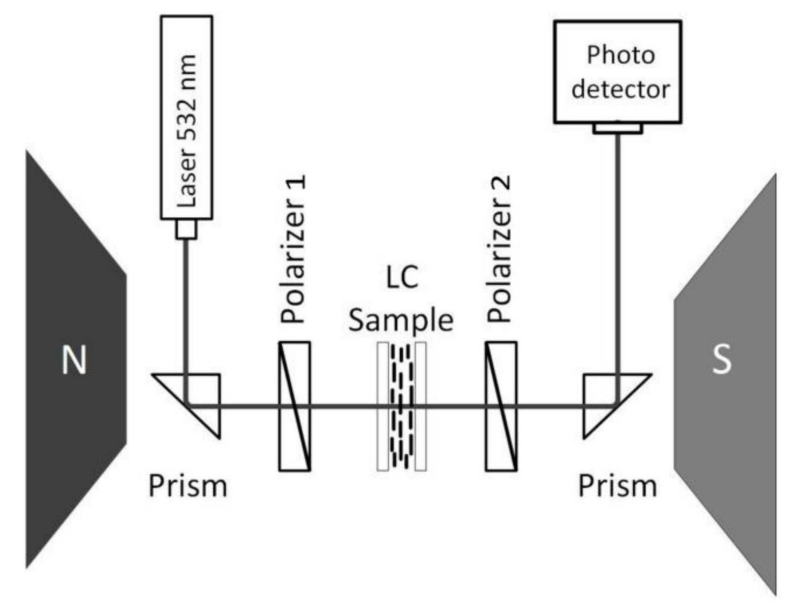

Fig. 1. Experimental arrangement for magneto-optical investigation.

\section{Results and discussion}

The magneto-optical behavior was investigated in the sets of ferronematic LCs doped with magnetic particles of different shape (size) and concentration using the experimental arrangement shown in Fig. 1. The initial position of the first polarizer, for all investigated suspensions ensured that the incident light beam was linearly polarized, whereas the LCs position provided for maximal transmittance registered by a photodetector, and the second polarizer was adjusted to obtain maximal transmittance after the partial depolarization caused by LC. 
Concerning the LC cell position, it could be rotated by about $90^{\circ}$ to measure the depolarization effect of investigated LCs when the magnetic field was applied. The light intensity as a function of magnetic field or time was then registered. Presented magneto-optical characteristics were also compared with characteristics obtained using crossed polarizers. These showed the same characteristics. However, the light intensity was much weaker.

Figure 2 shows the light transmission, expressed as $I / I_{0}$, as a function of magnetic field for $6 \mathrm{CHBT}$ liquid crystal doped with both spherical and chain-like nanoparticles. $I_{0}$ is the intensity of incident light passing through the LC cell without applied magnetic field. The main difference between these doped LCs is a transmission increasing overshoot in the case of chain-like particles. It was observed also when the magnetic field pulses higher than $275 \mathrm{mT}$ (decreasing region) were applied. However, the decrease of the light intensity to the minimum value relates to superimposed oscillations.

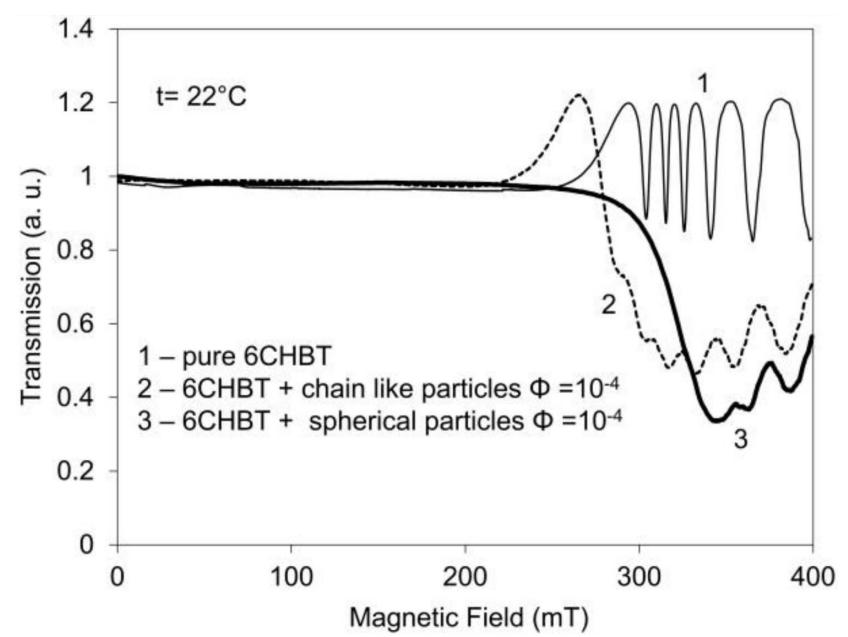

Fig. 2. Magneto-optical characteristics of pure 6CHBT liquid crystal (1), doped with chain-like (2) and spherical (3) magnetic particles $\left(\Phi=1 \times 10^{-4}\right)$.

It is assumed that during the lowered threshold field a planar aligned molecular director undergoes a reorientation between initial planar one and final perpendicular one and this process of reorientation is clearly revealed by the ellipticity variation. Therefore, when the LC is subjected to a laser beam, a succession of maxima and minima of the transmitted light through the LC cell appears [25]. The same situation occurs when the magnetic field is turned off and the LC returns to the initial planar orientation. These oscillations are present also on the curve (1) corresponding to the pure LC and indicate the fact that they are the peculiarity of LC molecules under magnetic field and illumination.

Figure 3 shows the magneto-optical time responses of the LC doped with chain-like magnetic particles when the magnetic field pulses of 250 and $400 \mathrm{mT}$ are applied. Observed responses correspond to magneto-optical characteristics (Fig. 2). While the magnetic field $B=$ $250 \mathrm{mT}$ causes the increase of light transmission, the magnetic field higher than $275 \mathrm{mT}$ causes its decrease but with a more complicated attribute. After increasing for $\approx 2 \mathrm{~s}$, the transmission decreases to its minimum, during which oscillations are superimposed. Then it increases to its equilibrium state. After the magnetic field is switched off the response is similar but opposite, characterized in sequence by oscillations, overshoot, and increase to the initial equilibrium. The initial state is then reached during next $\approx 40 \mathrm{~s}$. The similar response was observed in the case of spherical particles after the application of $400 \mathrm{mT}$ pulsed magnetic field, except for the increasing peak. The threshold field which is defined as the field where the light transmission is decreased to $90 \%$ of the initial value (zero field) [8], was present for both kinds of dopants close to $\approx 300 \mathrm{mT}$.

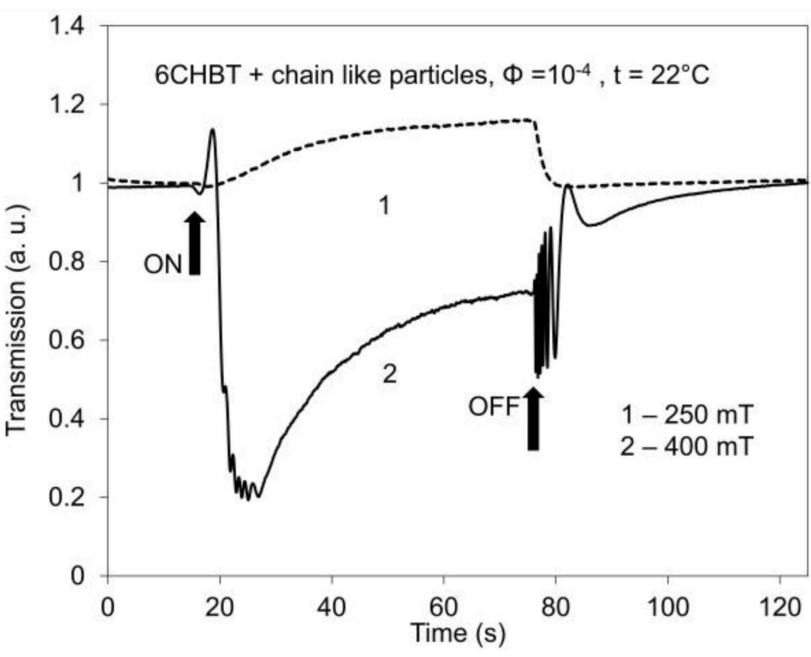

Fig. 3. Magneto-optical time response of 6CHBT liquid crystal doped with chain-like magnetic particles $\left(\Phi=1 \times 10^{-4}\right)$ after magnetic field pulses $B=$ $250 \mathrm{mT}$ (1) and $B=400 \mathrm{mT}(2)$ were applied.

Similar position of the threshold was also observed, except for the lowest concentration $\Phi=1 \times 10^{-5}$, for the LC doped with rod-like particles (Fig. 4). The position of the threshold field, however, is caused by the presence of ferroparticles and as it is attributed to the combination of both ferromagnetic and anchoring energies, it depends on both the concentration and shape of nanoparticles. The transmission in the case of highest concentration ( $\Phi=1 \times 10^{-4}$ ) of rod-like particles begins to change just after the magnetic field is applied. Such development can be attributed to the presence of different regimes of the threshold orientational behavior of compensated ferronematics under magnetic field [16, 26, 27]. The reason lies in successive magnetization of the ferronematic in the direction of the director, rotation of the director in the external field, and finally synchronous rotation of the director and magnetization of compensated ferronematics along the applied field. Similar development 
of magneto-optical characteristic was observed also in the case of 6CHBT doped with functionalized SWCNTs of the same concentration [28]. Although the obtained shift for the lowest concentration is not clear yet, similar behaviour, that is the non-continuous change of the threshold field with increasing/decreasing particle concentration, was observed also in other works (for example $[29,30])$. Some reason for this effect, however, could be the processes of aggregation that represent the actual fact about lowering of effective concentration of particles in the cases of higher concentration.

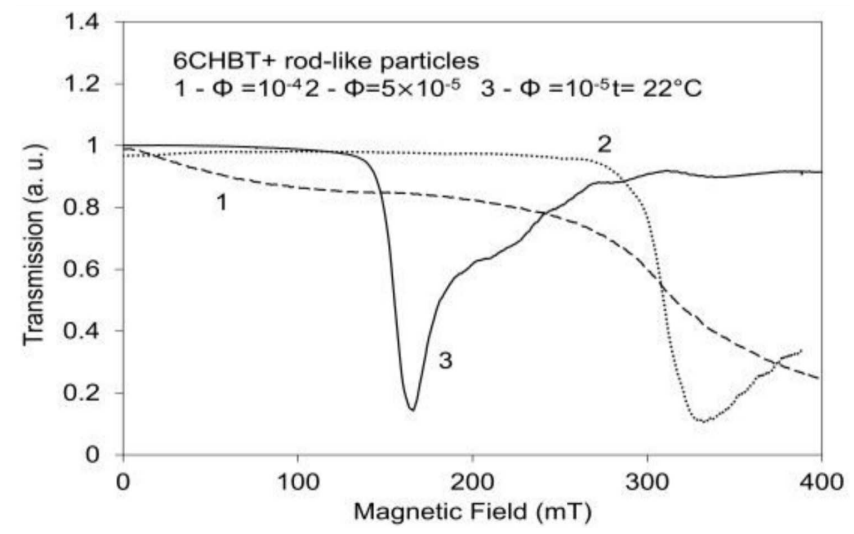

Fig. 4. Magneto-optical characteristics of 6CHBT liquid crystal doped with rod-like magnetic particles with concentrations $\Phi=1 \times 10^{-4}(1), \Phi=5 \times 10^{-5}(2)$ and $\Phi=1 \times 10^{-5}(3)$.

Oscillations, when the magnetic field is switched on or switched off, were observed in LCs doped with carbon nanotubes [31], and also when the electric field was applied $[8,30]$. They were observed also in LC layers situated on the polycarbonate surface under the magnetic field [11]. The origin of these oscillations thus can be attributed to the directional redistribution of LC molecules around the equilibrium position of the nematic director, from uniform (planar) alignment on the cell surfaces to the perpendicular at the center of the LC layer, during increase in magnetic field $[11,30]$. The shape of oscillation of a saturation regime observed during measurements of the phase difference in magnetically doped nematic LCs were taken as a part of their collective behavior [32]. The transmission oscillations are accordingly induced by a reorientation of the molecular director when the LC is subjected to an external field higher than the transition threshold and to a laser beam. The order number of transmission maxima and minima, occurring when the external field is higher than the threshold field, can be a function of the maximum deviation angle, the cell thickness and the light wavelength [31, 33]. Therefore, the number of observed transmission maxima can be lower $[11,17,34]$, but higher $[16,31]$ than in our magnetooptical experiments (five transmission maxima).

The role of magnetic field strength on the optical transmission is shown in Fig. 4 for three different concentrations of rod-like magnetic particles in investigated LC.
In contrast to previous characteristics (Fig. 2), no strong oscillations occur. Only in LCs doped with lower concentrations, very small indication of oscillation can be recognized. This fact can coincide with previous results presupposing the role of a process of particles aggregation $[16,35]$. The increase of the concentration, although, insures the stability of the curve. Magneto-optical time responses of the LC doped with rod-like magnetic particles of the same concentration, $\Phi=10^{-4}$, after magnetic field pulses of different magnitudes (100, 250, and $400 \mathrm{mT}$ ) were applied, as shown in Fig. 5. It is evident that the stronger anchoring in the case of rod-like particles (rigid anchoring) ensures stable magneto-optical responses. It should be noted that while capacitance measurements [23] showed weak anchoring only in the case of spherical magnetic particles and in the case of both chainlike and rod-like particles rigid anchoring, our SAW [1921] and presented magneto-optical measurements indicated similar behaviour for spherical and chain-like particles but different behaviour in the case of rod-like particles. This fact supports rather the conclusion that the rigid anchoring is above all the characteristic property of only rod-like magnetic particles in LC suspension.

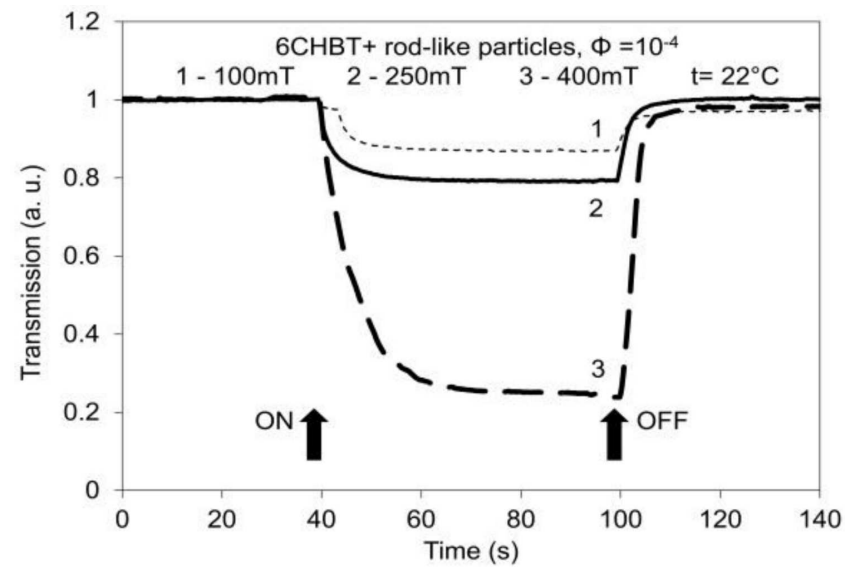

Fig. 5. Magneto-optical time responses of 6CHBT liquid crystal doped with rod-like magnetic particles $(\Phi=$ $1 \times 10^{-4}$ ) after magnetic field pulses $B=100 \mathrm{mT}(1)$, $B=250 \mathrm{mT}(2)$, and $B=400 \mathrm{mT}$ (3) were applied.

The registered partial depolarization was verified by transmission measurement when the LC cell was rotated by about $90^{\circ}$. Magneto-optical time responses, when the LC cell was rotated by about $90^{\circ}$, are shown for two different magnetic fields in Fig. 6. Observed responses, as compared to the previous ones (Fig. 5), show the increase of optical transmission that is, similar to the decrease in transmission in the original arrangement, as a result of partial depolarization (elliptical polarization) of the incident light beam by LC cell. The change of the correlative orientation between LC molecules and light polarization plane, as it can be seen, again gives rise to the existence of oscillations. The effect of partial depolarization in LCs including the pure 6CHBT liquid crystal was also 
already investigated $[35,36]$ and oscillations observed on ellipticity curves were attributed to the fact that during the Fréedericksz transition the director of planar alignment sample undergoes a reorientation between two fixed directions, from planar to normal [36].

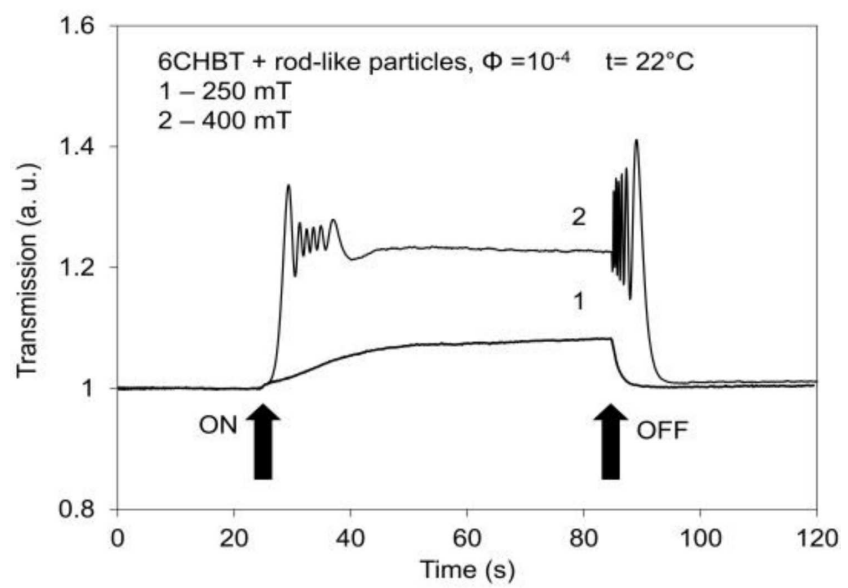

Fig. 6. Magneto-optical time responses of 6CHBT liquid crystal doped with rod-like magnetic particles $(\Phi=$ $\left.1 \times 10_{-4}\right)$ after magnetic fields $B=250 \mathrm{mT}(2)$ and $B=$ $400 \mathrm{mT}(3)$ were applied ( $\mathrm{LC}$ cell rotated about $90^{\circ}$ ).

The measurement at increasing/decreasing magnetic field, illustrated in Fig. 7, shows quite clearly the hysteresis in the region of decreasing light transmission. Similar hysteresis was obtained also for other particle concentrations. However, no memory effect was observed as it is clearly apparent also from Fig. 7, where it is evident that the transmission at the magnetic field decreasing to zero directs towards the initial value. The observed hysteresis also supports the assumption that at higher fields some process of magnetic particles aggregation occurs.

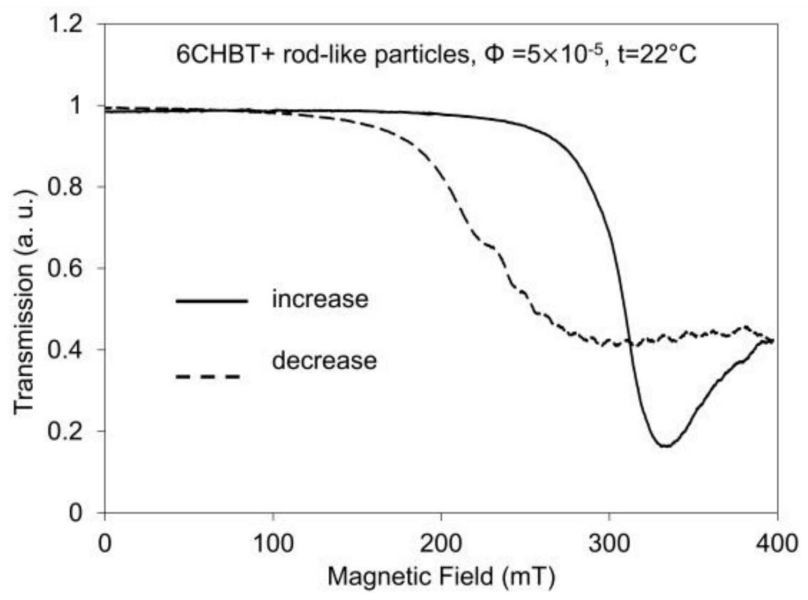

Fig. 7. Magneto-optical characteristics of 6CHBT liquid crystal doped with rod-like magnetic particles with concentrations $\Phi=5 \times 10^{-5}$ measured for increasing/decreasing magnetic field.
Figure 8 illustrates, for the comparison, magnetooptical time responses after the application of $60 \mathrm{~s}$ lasting pulse of the same magnetic field $(B=400 \mathrm{mT})$ and the same concentration $\left(\Phi=10^{-4}\right)$ for all dopants, including spherical, chain-like, and rod-like magnetic particles.

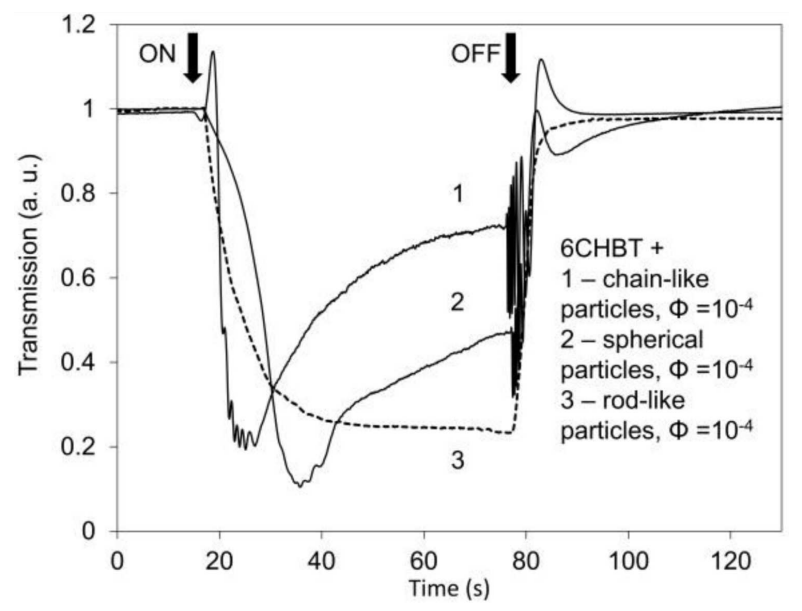

Fig. 8. Magneto-optical time responses of 6CHBT liquid crystal doped with rod-like magnetic particles $(\Phi=$ $1 \times 10^{-4}$ ) after magnetic fields $B=250 \mathrm{mT}(2)$ and $B=$ $400 \mathrm{mT}$ (3) were applied (LC cell rotated about $90^{\circ}$ ).

The comparison of all observed magneto-optical characteristics and magneto-optical time responses indicates that both concentration and shape (size) of magnetic particles play a very important role in the magneto-optical behavior. However, while on the one hand, the increase in both particle size and concentration gives rise to the stable response, on the other hand it extends the switchingon time. This behavior can be attributed to the higher stability of larger particles with regards to possible oscillations wherewith it can be connected also with the process of particles aggregation.

\section{Conclusions}

In this work, the magneto-optical behavior of the ferronematic liquid crystal 6CHBT doped with spherical, chain-like, and rod-like magnetic nanoparticles was investigated. The magneto-optical effect was examined using the initial linearly polarized laser beam $(532 \mathrm{~nm})$ passed through liquid crystal cell under continuously increasing and/or pulsed magnetic field. The results obtained that followed up with our previous investigations confirmed both previously supposed orientational coupling between the magnetic particles and the liquid crystal matrix and the improvement of optical properties including the switching behavior using the appropriate type of magnetic particles. Except for the marked decrease of the threshold field and stronger magneto-optical responses, the decrease of the oscillation amplitude and the resulting higher stability of magneto-optical responses were achieved by increasing of both amount and size 
of magnetic particles in LCs, althought at the expense of the extension of the switching-on time. Presented magneto-optical measurements also supported the conclusion that the rigid anchoring is above all the characteristic property of rod-like magnetic particles only in LC suspension.

\section{Acknowledgments}

This work was supported by the VEGA project 2/0016/17 and the project of Slovak Research and Development Agency under the contract No. APVV- 349 015-0453. Authors also would like to thank Mr. František Cernobila for technical assistance.

\section{References}

[1] F. Brochard, P.G.D. Gennes, J. Phys. (France) 31, 691 (1970).

[2] J. Liebert, A. Martinet, J. Phys. Lett. 40, 363 (1979).

[3] S.V. Burylov, Y.L. Raikher, I. Extend. Contin. Model Mol. Cryst. Liq. Cryst. 258, 107 (1995).

[4] S.V. Burylov, Y.L. Raikher, Mol. Cryst. Liq. Cryst. 258, 123 (1995).

[5] N. Tomašovičová, M. Timko, Z. Mitróová, M. Koneracká, M. Rajňak, N. Éber, T. Tóth-Katona, X. Chaud, J. Jadzyn, P. Kopčanský, Phys. Rev. E 87, 014501 (2013).

[6] P.G. Gennes, The Physics of Liquid Crystals, Clarendon Press, Oxford 1974.

[7] V.I. Zadorozhnii, A.N. Vasilev, V.Y. Roshentnyak, K.S. Thomas, T.J. Sluckin, Europhys. Lett. 73, 408 (2006).

[8] W. Lee, C.Y. Wang, Y.C. Shih, Appl. Phys. Lett. 85, 513 (2004).

[9] G. Cook, V.Yu. Reshetnyak, R.F. Ziolo, S.A. Basun, P.P. Banerjee, D.R. Evans, Opt. Expr. 18, 17339 (2010).

[10] G. Si, Y. Zhao, E.S.P. Leong, Y.J. Liu, Liq.-Cryst.Enabled Act. Plasmon. Mater. 7, 1296 (2014).

[11] A.M. Prshin, V.A. Gunyakov, V.Y. Zyryanov, V.F. Shabanov, Int. J. Mol. Sci. 15, 17838 (2014).

[12] V.S. Sutorin, M.N. Krakhalev, O.O. Prischepa, V.Y. Zyryanov, JETP 96, 511 (2012).

[13] P.R.G. Fernandes, H. Mukai, I.M. Laczkowski, J. Magn. Magn. Mater. 289, 115 (2015).

[14] H. Mukai, S.M. Shibli, P.R.G. Fernandes, J. Mol. Liq. 135, 53 (2007).
[15] C. Rosu, C. Cartoaje, A.L. Paun, Optoelectron. Adv. Mater.-Rapid Commun. 3, 127 (2009).

[16] A. Mertelj, D. Lisjak, Liq. Cryst. Rev. 5, 1 (2017).

[17] N. Podoliak, O. Buchnev, O. Buluy, G. D'Alessandro, M. Kaczmarek, Y. Reznikov, T.J. Sluckin, Soft Matter 7, 4742 (2011).

[18] P.M. Rupnik, D. Lisjak, M. Čopič, A. Mertelj, Liq. Cryst. 42, 1684 (2015).

[19] P. Bury, J. Kúdelčík, Š. Hardoň, M. Veveričík, P. Kopčanský, M. Timko, V. Závišová, J. Magn. Magn. Mater. 423, 57 (2017).

[20] P. Bury, M. Veveričík, J. Kúdelčík, P. Kopčanský, M. Timko, V. Závišová, Acta Phys. Pol. A 131, 913 (2017).

[21] P. Bury, M. Veveričík, J. Kúdelčík, P. Kopčanský, M. Timko, V. Závišová, Acta Acust. united with Acustica 104, 48 (2018).

[22] P. Kopčanský, N. Tomašovičová, T. Tóth-Katona, N. Éber, M. Timko, V. Závišová, J. Majorošová, M. Rajňak, J. Jadzyn, X. Chaud, Magnetohydrodynamics 49, 586 (2013).

[23] P. Kopčanský, N. Tomašovičová, M. Koneracká, V. Závišová, M. Timko, A. Džárová, A. Šprincová, Phys. Rev. E 78, 011702 (2008).

[24] N. Tomašovičová, P. Kopčanský, M. Koneracká, V. Závišová, M. Timko, N. Éber, T. Tóth-Katona, J. Jadzyn, J. Phys. Condens. Matter 20, 204123 (2008).

[25] S.V. Burylov, Y.L. Raikher, J. Magn. Magn. Mater. 122, 62 (1993).

[26] A.N. Zakhlevnykh, D.A. Petrov, Phase Transit. 87, 1 (2013)

[27] A.N. Zakhlevnykh, D.A. Petrov, J. Magn. Magn. Mater. 401, 188 (2016).

[28] P. Bury, M. Veveričík, P. Kopčanský, M. Timko, Z. Mitróová, Physica E 103, 53 (2018).

[29] C. Vena, C. Versace, G. Strangi, S. D‘Elia, R. Bartolino, Opt. Expr. 15, 17063 (2007).

[30] P. Porov, V.S. Chandel, J. Sci. Arts 16, 249 (2016).

[31] C. Cirtoaje, E. Petrescu, Physica E 84, 244 (2016).

[32] S.H. Chen, N.M. Amer, Phys. Rev. Lett. 51, 2298 (1983).

[33] A.N. Zakhlevnykh, J. Magn. Magn. Mater. 269, 238 (2004).

[34] S.Y. Lu, L.C. Chien, Opt. Expr. 16, 12777 (2008).

[35] P.V.D. Schoot, J. Chem. Phys. 117, 3537 (2002).

[36] A.W. Domanski, D. Budaszewski, M. Sierakowski, T.R. Wolinski, Opto-electron. Rev. 14, 305 (2006). 